\title{
Philosophiques
}

\section{Précis de L’adresse du réel}

\section{Jocelyn Benoist}

Volume 45, numéro 1, printemps 2018

URI : https://id.erudit.org/iderudit/1048622ar

DOI : https://doi.org/10.7202/1048622ar

Aller au sommaire du numéro

Éditeur(s)

Société de philosophie du Québec

ISSN

0316-2923 (imprimé)

1492-1391 (numérique)

Découvrir la revue

Citer ce document

Benoist, J. (2018). Précis de L'adresse du réel . Philosophiques, 45(1), 215-221.

https://doi.org/10.7202/1048622ar

Ce document est protégé par la loi sur le droit d'auteur. L'utilisation des services d'Érudit (y compris la reproduction) est assujettie à sa politique d'utilisation que vous pouvez consulter en ligne.

https://apropos.erudit.org/fr/usagers/politique-dutilisation/
Cet article est diffusé et préservé par Érudit.

Érudit est un consortium interuniversitaire sans but lucratif composé de l’Université de Montréal, l'Université Laval et l'Université du Québec à Montréal. Il a pour mission la promotion et la valorisation de la recherche. https://www.erudit.org/fr/ 


\title{
Disputatio
}

\section{Précis de L'adresse du réel'}

\author{
JOCELYN BENOIST
}

Université Paris 1 Panthéon-Sorbonne

ISJPS

La finalité de ce livre est double. D’un côté, il formule une prise de position dans l'espace des discussions suscitées par l'apparition des «nouveaux réalismes" sur la scène philosophique européenne et, au-delà, internationale. On y trouve donc, par exemple, une présentation et des éléments de discussion des travaux de Markus Gabriel, Maurizio Ferraris et, dans une moindre mesure, Quentin Meillassoux. Par rapport à ces différentes recherches et voies philosophiques, j'essaie de cerner des points d'accord et de désaccord, tentant ainsi au fond de construire le champ des réalismes contemporains, pour essayer de produire une intelligibilité du retour de ce motif, au-delà des frontières qui fragmentent le monde philosophique d'aujourd'hui — notamment la division entre philosophies respectivement dites «continentale» et "analytique». De l'autre côté, il s'agit aussi et d'abord pour moi d'approfondir et prolonger les réflexions ouvertes dans mon livre Eléments de philosophie réaliste (Paris, Vrin, 20II), en direction de ma forme propre de réalisme ou en tout cas de ma façon propre de poser la question du réalisme, suivant une orientation et une méthodologie marquées, essentiellement, par l'influence de Wittgenstein.

Un mot sur le titre ne sera pas inutile, car il peut bien résumer le propos de l'ouvrage. L'idée générale de L'adresse du réel, c'est précisément qu'il n'y a pas de telle "adresse »: il n'y a pas de sens à vouloir chercher le réel dans un genre particulier de choses au détriment d'autres genres. Cela pour deux raisons: parce qu'il n'y a pas de question du réel in abstracto, mais le réel est toujours ce qui se distingue de ce qui ne l'est pas là où un problème déterminé, une question, est posée à la réalité, ou à propos d'elle; mais aussi parce qu'en prétendant isoler des genres de choses qui seraient $a$ priori réelles - et constitueraient «le réel» — on risque par là même d'accréditer qu'il existe, à côté des genres de choses intrinsèquement réelles, des genres de choses qui ne le seraient pas: pour ainsi dire des «choses irréelles ». Or l'intuition de base du réalisme est qu'il n'y a pas de telles «choses» ou, ce qui revient au même, qu'elles ne constituent pas un genre. L'irréel, si en tout cas on doit lui prêter quelque force de négation que ce soit du réel, ne constitue pas réellement un autre genre d'être à côté du réel: ce sont alors

1 Jocelyn Benoist, L'adresse du réel, Paris, Vrin, collection "Moments philosophiques ", 20I7, 376 pages. La revue Philosophiques remercie Maxime Doyon pour la coordination de cette disputatio.

PHILOSOPHIQUES 45/1 — Printemps 2018, p. 215-221 
exactement le même genre de choses qui, suivant les circonstances, peuvent être réelles ou non réelles. Il n'y a donc, deux fois, pas de sens à attribuer le monopole de la réalité à un certain genre ou à de certains genres $a$ priori.

Même si ce n'est pas dit dans ce livre - mais l'idée était présente dans le précédent Logique du phénomène (Paris, Éd. Hermann, 2016) "l'adresse", inversement, pourrait ici renvoyer positivement au fait que le réel est essentiellement objet d' "adresse » : le réel est ce qui ne peut en aucun cas se privatiser, et il n'est de réel que pour les autres - et non pour moi seul. D'où l'importance du fait que le réel comme tel ait, essentiellement, à être dit, et de la recherche, en situation, de la façon adéquate de le faire. En ce sens, «le réel » en général n'a pas d'adresse, mais il n'est de réel qu'adressé, et les explorations grammaticales de ce livre ne sont rien d'autre qu'autant de variations sur différentes façons de l'adresser et les enjeux qu'il peut y avoir à l'adresser.

Une dernière signification enfin pourrait être retenue: celle suggérée par Sandra Laugier dans un exposé à propos de mon livre, qui est cohérente en effet avec tout un aspect du propos du livre, suivant laquelle il y aurait toujours besoin d' " adresse ", c'est-à-dire d'habileté ainsi que de justesse, là où il y va du réel. Dans la mesure où les réflexions présentées dans ce livre relèvent bien d'une pensée de la phronésis et en un sens consistent à étendre ce modèle originairement pratique au champ théorique, selon une problématique du jugement, une telle interprétation paraîtra pertinente. Évidemment - et là peut-être s'arrête mon accord avec Sandra Laugier — je ne suis pas sûr que le réel s'identifie exactement à cette adresse qu'il requiert de notre part pour y faire droit. Catégorialement, je suis même tout à fait convaincu du contraire. Cette "adresse" n'en constitue pas moins une dimension extrêmement importante du sens du réel.

Dans le chapitre I, «Le réalisme sans la métaphysique », qui n'est peutêtre pas très bien nommé, mais dont le titre indique néanmoins quelque chose, je renvoie dos-à-dos un certain type de réalisme métaphysique, absolutiste, qui prétend se constituer en théorie, fût-elle minimaliste, d'un réel abstrait et décontextualisé, et sa contrepartie relativiste, qui n'est qu'un absolutisme négatif. Je distingue le relativisme, qui précisément consiste à admettre des contenus à valeur de vérité flottante et le contextualisme, qui consiste à restituer aux contenus leur finesse de grain en tenant compte de leur dépendance par rapport à la situation dans laquelle le réel est abordé. Absolutisme et relativisme font fond sur la même indépendance supposée du contenu par rapport à la réalité, installant la pensée hors-sol et s'opposant simplement dans leur façon respective de traiter cette entité comme intrinsèquement pourvue de valeur de vérité ou au contraire comme indéterminée à cet égard et donc soumise, dans sa vérité ou sa fausseté, aux aléas de la subjectivité ou toute autre forme de relativité. J'attaque la racine même de cette symétrie en remettant en question l'immunité du contenu par rapport aux usages réels et en allant chercher dans ces usages, en contexte, le prin- 
cipe même de la norme de vérité dans sa capacité à gouverner la prise de nos pensées et de nos énoncés sur le réel - et non sur leur simple image spéculaire, qu'on l'appelle «objet» ou autrement. Ce n'est qu'en réancrant les porteurs de vérité dans le réel qu'on pourra comprendre comment ils peuvent eux-mêmes se rapporter au réel.

Dans le chapitre II, «Nouveau(x) Réalisme(s)", j'ouvre un dialogue frontal avec les deux principaux acteurs du courant connu, depuis le manifeste du même nom², comme "Nouveau Réalisme", Markus Gabriel et Maurizio Ferraris, tout en esquissant une histoire du retour de ce motif réaliste dans la philosophie d'aujourd'hui, contre les constructivismes et relativismes linguistiques qui ont pu dominer dans les générations précédentes. Je souligne les aspects contextualistes de la démarche de Markus Gabriel et relève notre accord de principe sur ce point, tout en soulevant la question de la différence entre "être intentionnel » et «être réel », qui peutêtre nous écarte. Pour moi, une forme déterminée d'être intentionnel ne peut pas constituer un champ de sens autre que celui dans lequel un certain type d'être réel peut exister, car il n'est d'être intentionnel que rapporté comme norme à un être réel, dont il donne une mesure, et dans un champ de sens réel. Il est probable, à ce niveau, que Markus Gabriel et moi-même ayons des conceptions différentes de l'intentionalité. De l'autre côté, je discute le réalisme social de Maurizio Ferraris qui, dans une certaine phase au moins ${ }^{3}$, me semble mettre en œuvre une problématique constructionniste qui pourrait bien être une forme d'intentionalisme larvé. Là -contre, je mets en avant le caractère non seulement intentionnel - mais réel - de "la réalité sociale ", comme de toute réalité en général. Ce chapitre est donc placé globalement sous le signe de la critique de l'intentionalisme et de sa conséquence indésirable: les ontologies intentionnelles - ce qui ne signifie bien sûr pas l'élimination de toute idée d'intentionalité, mais un effort pour resituer cette structure (que je tiens pour définie par sa fonction normative) là où seulement elle a un sens: dans la réalité.

Le chapitre III approfondit le dialogue avec Markus Gabriel en abordant la question du statut de la notion de "sens", centrale dans son ontologie des "champs de sens", par rapport à la réalité. J'adhère au désir de réengagement ontologique du sens qu'on trouve certainement à la source de la thèse, anti-frégéenne, qu'il défend, selon laquelle les différents sens seraient des "propriétés des choses elles-mêmes". Pour des raisons probablement un peu différentes des siennes, l'idée d'entité intermédiaire, à plus forte raison pourvue d'une énigmatique capacité représentationnelle, comme

2. Maurizio Ferraris, Manifesto del nuovo realismo, Rome, Laterza, 20I2; trad. fr. Manifeste du nouveau réalisme, Paris, Hermann, 2014.

3. Mes remarques critiques se rapportent en premier lieu au Manifeste du nouveau réalisme. Je pense qu'elles seraient moins directement pertinentes pour Mobilisation totale, Paris, P.U.F., 2016. 
le «sens» frégéen (Sinn) selon au moins une certaine lecture, ne me plaît pas. Je ne saurais pour autant être d'accord pour reverser le "sens" frégéen, ou quelque version post-frégéenne dudit sens, du côté, purement ontologique, des propriétés de la chose même. En effet, je crois qu'il est essentiel de maintenir l'écart normatif entre la chose et ce selon quoi elle est déterminée dans une attitude de connaissance, ou peut-être, plus largement, de pensée. Perdre cet écart, c'est, en un sens, perdre le sens de la réalité. Aux fins de sa formalisation, il ne me semble pas inutile de maintenir la distinction entre "sens" et «réalité » en tant que distinction catégoriale — et non de deux "genres» d'être. Pour être tout à fait clair, la différence que j'essaie de mettre en avant dans ce chapitre comme dans d'autres est celle entre "vrai» et "réel ». Il ne me semble pas que ces deux déterminations soient du même ordre ni ne s'appliquent au même genre de choses. Je pense qu'il est important de distinguer logiquement ce qui relève de l'être et ce qui articule une vue sur l'être. A cet égard, je crois devoir me dissocier de l'analyse de la perception offerte par Markus Gabriel, dont je me sens pourtant très proche sur un autre plan. Je suis d'accord avec lui quant à la facticité - donc, dans mes termes, la réalité — de ce que nous appelons «illusion» dans le cas des illusions perceptuelles, qui, en un sens, sont tout juste des perceptions comme les autres. Cependant, je ne partage pas sa conception de la perception comme point de vue sur l'objet. Je place quant à moi le perçu plutôt du côté de la réalité tout simplement - il en a la facticité — et je considère qu'il ne peut devenir vue sur l'objet que là où précisément on le mesure à cette norme qu'est l'idée d'un certain objet, donc là où la réalité - y compris la réalité perçue - est interrogée du point de vue de la vérité de ce qu'on peut en dire ou en penser. Les aspects sensibles sont certainement, en effet, des propriétés de la chose perçue; mais, catégorialement, ce ne sont pas des "sens" au sens de Frege, ou en quelque sens adapté que ce soit, qui s'y rapporteraient. De tels «sens » peuvent assurément incorporer certaines dimensions de ces propriétés, en faire usage pour construire une prise logique sur la réalité, faite par là même «objet», mais, logiquement, ils ne se confondent jamais avec elles. Il faut, en ces matières comme ailleurs, maintenir le gouffre logique entre l'être et sa représentation. À quoi servirait donc la représentation, si elle se confondait avec ce qu'elle représente ou en devenait simplement un aspect ?

Dans le chapitre IV, «Rien que de la grammaire », je reviens au soubassement wittgensteinien de mon propos. J'affronte le soupçon classique d' "idéalisme linguistique» pesant sur les investigations grammaticales c'est-à-dire sur ce qui est dit — du genre de celles développées dans ce livre. À cette fin, j'entre en discussion avec l'article classique d'Elizabeth Anscombe $^{4}$, qui, à l'étude, s'avère extrêmement difficile. Mettre en avant,

4. Cf. Elizabeth Anscombe, «The Question of Linguistic Idealism», repris dans From Parmenides to Wittgenstein. The Collected Philosophical Papers of G.E.M. Anscombe, vol. I, Oxford, Blackwell, I98 I, p. I I2-I33. 
comme le fait une analyse wittgensteinienne, l'arbitraire de la grammaire, n'est-ce pas courir le risque de faire du langage un empire dans un empire et de le détacher du monde ou tout au moins - s'il faut critiquer le concept de «monde» — de la réalité ? Commentant la fameuse formule du $\mathbb{S} 37$ I des Recherches philosophiques suivant laquelle "l'essence est exprimée dans la grammaire ${ }^{5}$ ", en lui restituant sa véritable syntaxe, je montre comment elle fraie un chemin entre l'illusion transcendantale d'un langage dont les structures seraient les «reflets » de supposées structures intrinsèques de la réalité et l'illusion symétrique d'un langage complètement désengagé par rapport à celle-ci et l'organisant pour ainsi dire de l'extérieur. Méditant la formule suivant laquelle la grammaire, ce serait «les livres de compte du langage ${ }^{6}$, je mets en évidence ce en quoi l'absence de vérité de la grammaire ne doit pas faire perdre de vue qu'elle est, essentiellement, grammaire de la vérité, et qu'il n'est d'autre lieu pour elle que des transactions effectives dont l'enjeu est justement la réalité.

Dans le chapitre V, «Les frontières du réel », je défends précisément une conception grammaticale de l'impossible, visant à nous guérir de nos tentations philosophiques de le traiter comme un pseudo-domaine ontologique ou méta-ontologique. Après avoir, au chapitre II, dissous la sphère imaginaire de l'inexistant en tant que pseudo-sphère ontologique, je dégonfle maintenant la mythologie d'une sphère encore extérieure à celle de l'inexistant, qui serait celle de l'intrinsèquement impossible (c'est-à-dire de ce qui, a priori, ne pourrait pas exister). J'avance que l'impossible a priori n'a pas de consistance ontologique ou méta-ontologique propre, mais renvoie toujours à une mesure de ce qui est possible ou impossible, par définition appliquée à la réalité. Il n'y a donc pas de sens à chercher dans l'impossible un gisement d'entités - pas plus que de sens — au-delà du réel.

Dans le chapitre VI, «Requiem - tintamarre - pour une phénoménologie », je critique l'irréalisme de la phénoménologie transcendantale, qui poursuit une opération (de mise en suspens du jugement) au-delà du terrain et des limites, réelles, où celle-ci a un sens, et qui, de ce point de vue, effectue un geste agrammatical - ou en tout cas qu'elle n'arrive pas à regrammaticaliser de façon convaincante, installant son propos dans une fondamentale équivocité. Contre la figure husserlienne de la réduction transcendantale, je mets en avant la pratique d'une réduction réelle, qui a précisément pour portée de révéler le réel, dont je trouve l'exemple dans le dispositif acousmatique, tel qu'il fut mis en œuvre par Pierre Schaeffer — même si je m'éloigne

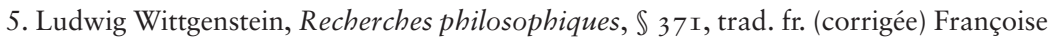
Dastur, Maurice Elie, Jean-Luc Gautero, Dominique Janicaud, Elisabeth Rigal, Paris, Gallimard, 2004, p. 170.

6. Ludwig Wittgenstein, Grammaire philosophique, trad. fr., trad. fr. Marie-Anne Lescourret, Paris, Gallimard, I980, p. II9. 
de la théorisation, résiduellement et à mon avis improprement phénoménologique, qu'il en propose.

Le chapitre VII poursuit la déphénoménologisation du sensible ainsi entamée dans le chapitre VI, en faisant ressortir la puissance propre de réalité du sensible. Il le fait en passant sur le terrain de la question philosophique classique de l'hallucination. Prolongeant et corrigeant dans une certaine mesure les analyses proposées dans un livre précédent $^{7}$, il défend l'idée que conjonctivisme pas plus que disjonctivisme ne fournissent un cadre adéquat pour rendre compte de l'hallucination, qui est une perception et qui, en tant que perception, a la puissance de réalité qui est celle de toute perception - en d'autres termes: est une réalité. Ces théories qui se partagent aujourd'hui la scène en philosophie de la perception (mais elles relèvent en réalité plutôt de l'épistémologie) sont l'une et l'autre également sujettes à cette même erreur qui consiste à aborder la perception d'un point de vue exclusivement épistémique et à mesurer l'expérience perceptuelle à une norme cognitive qu'elles lui imposent prématurément en ignorant sa facticité, à laquelle l'hallucination n'est pas une exception, mais dont elle constitue au contraire un exemple éminent. Ainsi, elles manquent la réalité du perçu, au double sens de: I) la teneur particulière de sa réalité (son contenu et ses modalités d'expérience propres); et 2) le simple fait que, intrinsèquement, il soit réel.

Le chapitre VIII prolonge ces réflexions sur la puissance de réalité du perçu. Revenant sur le débat entre Charles Travis et John McDowell, il met en évidence le caractère catégorialement biface du concept de "perception ", qui d'un côté renvoie à un fait, et de l'autre à un accomplissement normatif (de type cognitif). Je fais ressortir la facticité du perçu, généralement manquée par les analyses épistémiques de la perception, ou mésinterprétée au sens d'une théorie de l'évidence. La facticité du perçu n'est bien sûr pas synonyme de vérité. Ces deux termes appartiennent à des catégories différentes, et c'est un trait constitutif d'un point de vue réaliste d'apprendre à les distinguer. Pour cette même raison, la distinction entre sens épistémique (cognitif) et sens facticiel de la perception n'implique, en tant que distinction catégoriale, aucune opacité particulière de l'un à l'autre. C'est catégorialement que le perçu, en un certain sens du terme, n'est pas «conceptuel », ce qui ne veut pas dire qu'il soit "non conceptuel» au sens dans lequel ce terme est généralement employé dans les débats contemporains en tant que débats purement épistémologiques. Il n'y a pas de «contenu non conceptuel» et le perçu, au sens où, constitutivement, il n'est pas conceptuel, n'est pas un "contenu» au sens donné à ce terme par l'épistémologie moderne. Cependant, en rester au point de vue épistémique, qui est celui du «contenu », c'est, philosophiquement, manquer dans une très large mesure la percep-

7. Jocelyn Benoist, Le bruit du sensible, Paris, Éd. du Cerf, 20I3, chap. iii. 
tion: ignorer le sensible comme tel, qui désigne une dimension ontologique, et non épistémique.

Dans le chapitre IX, «La nature poétique du sensible», dans une forte continuité avec mon livre Le bruit du sensible, j'esquisse une réflexion sur les conditions de ce qui n'est plus une manifestation (suivant l'axe erroné être/apparence) mais une révélation de cette réalité qui est celle du sensible. À cette révélation, certaines opérations sont nécessaires. J'en trouve le modèle dans l'art en général, et certaines pratiques artistiques en particulier. La musique de Scelsi, subvertissant la norme instituée et définissant une nouvelle prise sur la réalité du son devient paradigmatique d'une telle procédure révélatoire. Le sensible, en tant que réalité — ce qui ne veut pas dire, soit dit en passant, qu'il constitue le tout de la réalité — ressort ici dans son statut de ressource, et non de représenté, de matière (Stoff) et non d'objet.

Il serait erroné de penser que le chapitre $\mathrm{X}$, "Qu'est-ce qu'être réaliste en morale?", représente une simple application des analyses développées par ailleurs dans mon livre au champ de la philosophie morale, ou d'ailleurs une véritable prise de position doctrinale dans ce champ. Il ne s'agit évidemment pas de mon domaine d'expertise, et mon propos n'est pas de défendre une doctrine particulière en philosophie morale. Le sens de ce chapitre, qui réfléchit sur des exemples empruntés à l'Ulysse de Joyce et au récit de Marta Hillers Une femme à Berlin, est plutôt que le passage sur le terrain moral, outre qu'il est rendu nécessaire par la perspective pluraliste et multidimensionnelle adoptée par le livre eu égard au concept de réalité, éclaire quelque chose de la nature de ce réalisme, qui refuse tout sens prédéterminé de "la réalité » : à savoir précisément que ce sens est, en chaque situation, à déterminer, et qu'une telle détermination met en jeu, à chaque fois, un art $d u$ jugement, qui implique nécessairement une responsabilité. Point évidemment particulièrement manifeste sur le terrain moral et qui, en un certain sens, pourrait lui donner valeur exemplaire pour ce réalisme. 